\title{
X-ray Dim Isolated Neutron Stars and phase-dependent absorption features
}

\author{
A. Borghese ${ }^{1}$, N. Rea ${ }^{1,2}$ and F. Coti Zelati ${ }^{2}$ \\ ${ }^{1}$ Anton Pannekoek Institute for Astronomy, \\ University of Amsterdam, Amsterdam, the Netherlands \\ email: a.borghese@uva.nl \\ ${ }^{2}$ Institute of Space Sciences (IEEC-CSIC), Campus UAB, \\ Barcelona, Spain
}

\begin{abstract}
Thanks to a detailed pulse phase spectroscopy of archival XMM-Newton observations, we discovered narrow and strongly phase-dependent absorption features in the X-ray spectra of two X-ray dim isolated neutron stars (XDINSs), RX J0720.4-3125 and RX J1308.6+2127. The spectral lines show similar properties: they are detected in only $20 \%$ of the rotational cycle with an energy of $\sim 740 \mathrm{eV}$ and appear to be stable over the timespan covered by the observations. The strong dependence on the pulsar rotation and the narrow width suggest that the features are most likely due to proton cyclotron resonant scattering in a confined magnetic structure close to the stellar surface. The inferred magnetic field in such a loop is of the order of $\sim 10^{14} \mathrm{G}$, higher than the surface dipolar magnetic field $\left(\sim 1-3.5 \times 10^{13} \mathrm{G}\right.$ for the XDINSs).
\end{abstract}

Keywords. X-rays: stars, stars: neutron, stars: individual (RX J0720.4-3125,RX J1308.6+2127)

\section{Introduction}

RX J0720.4-3125 and RX J1308.6+2127 are part of a group of seven nearby ( $\lesssim 500 \mathrm{pc})$, radio-quiet isolated neutron stars, referred to as the X-ray dim isolated neutron stars (XDINSs). They are characterized by X-ray luminosity of $10^{31-32} \mathrm{erg} \mathrm{s}^{-1}$ and relatively long periods $(P \sim 3-12 \mathrm{~s})$. XDINSs slow down with period derivatives of the order of $10^{-14}-10^{-15} \mathrm{~s} \mathrm{~s}^{-1}$ and have inferred surface dipolar fields of $\sim 10^{13} \mathrm{G}$ (see Turolla 2009 for a review). The XDINS X-ray spectra show soft $(0.2-1.2 \mathrm{keV})$ quasi-thermal emission with blackbody temperatures in the range of $50-100 \mathrm{eV}$ and six of them exhibit broad absorption features. The origin of these features is still unclear: they might be due to proton cyclotron resonances/atomic transitions in a magnetized atmosphere or to an inhomogeneous surface temperature distribution.

Recently, phase-dependent features have been reported in two low-field magnetars, SGR 0418+5729 and Swift J1822.3-1606 (Tiengo et al. 2013; Rodríguez Castillo et al. 2016). These discoveries have motived our search for similar features in the XDINS spectra. According to the most up-to-date magneto-thermal evolution models XDINSs are considered to be aged magnetars, therefore we expect to observe such phase-dependent features in their X-ray spectra.

\section{Results}

We re-analysed all the archival XMM-Newton observations for the XDINSs in search for phase-dependent spectral features. We found two possible candidates in RX J0720.43125 and RX J1308.6+2127 and derived upper limits for all the other XDINSs (for details about the data reduction and spectral analysis see Borghese et al. 2017, 2015).

Firstly, we built normalized energy versus phase images binning the source counts in rotational phase and energy channels (see fig. 1). This allowed us to look for spectral 


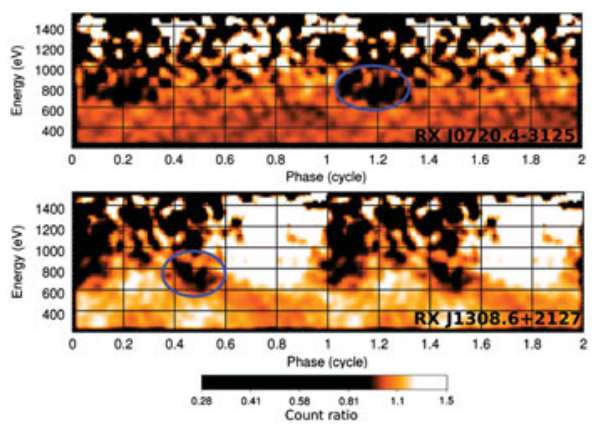

Figure 1. Normalized energy versus phase images for the longest observation of RX J0720.4-3125 (top, 51 ks) and RX J1308.6+2127 (bottom, $35.1 \mathrm{ks)} \mathrm{obtained}$ by binning EPIC pn source counts into 100 rotational phase bins and $25-\mathrm{eV}$-wide energy channels. The circles mark the phase-dependent features. Two cycles are shown for better visualization.

features that vary along the phase without making assumptions about the spectral energy distribution. The images in figure 1 show the features (marked with a circle) for RX J0720.4-3125 (top panel) and RX J1308.6+2127 (bottom panel); they are produced by a lack of counts with respect to the nearby energy channels. This gave us hints for spectral lines that are present in only a limited phase interval. As a next step we performed a detailed pulse phase spectroscopy by dividing the rotational phase in different bins and extracting the corresponding spectra. We fitted each of the phase-resolved spectra with the spectral model of the phase-averaged spectra (the null model) and for some of them we observed a discrepancy between the data and the model. Therefore we included a Gaussian line in absorption (the alternative model) that led to an improvement in the residual shape. For RX J0720.4-3125 and RX J1308.6+2127 the phase-dependent spectral lines have an energy of $\sim 740 \mathrm{eV}$, a width consistent with the energy spectral resolution of the EPIC pn camera and equivalent width of $\sim 30$ and $15 \mathrm{eV}$, respectively. To test the significance of the line, we performed Monte Carlo simulations: we simulated $10^{5}$ spectra according to the null model and fitted them with the alternative model. None of the simulated spectra show an equivalent width greater than the observed values, this means that the probability of the features being a statistical fluctuation is $<10^{-5}$. These simulations support our discoveries with a confidence level $>4.6 \sigma$ (for further details see Borghese et al. 2017, 2015).

\section{Discussion}

The similarities (strong dependence on the rotational phase and narrow width) between the features found in the low-field magnetars and the XDINSs suggest that the same mechanism could work: they might be explained by proton cyclotron resonant scattering. This scenario naturally accounts for the sharp variation with phase if there are smallscale magnetic structures close to the stellar surface. From the line energy, the magnetic field strength of these loops is of the order of $10^{14} \mathrm{G}$, about one order of magnitude for both sources.

These findings strengthen the evolutionary link between magnetars and XDINSs, thought to be aged magnetars. Moreover, according to this interpretation, the magnetic field of highly magnetized neutron stars is complex with deviations from a pure dipole, such as confined high $B$-field bundles.

\section{References}

Borghese A., Rea, N., Coti Zelati F. et al. 2015, ApJ, 807, L20

Borghese, A., Rea, N., Coti Zelati F. et al. 2017, MNRAS, 468, 2975

Rodríguez Castillo, G. A.,Israel G. L., Tiengo A. et al. 2016, MNRAS, 456, 4145

Tiengo, A., Eposito P., Mereghetti S. et al. 2013, Nature, 500, 312

Turolla, R. 2009, Astrophysics and Space Science Library, 357, 141 